\title{
ZWINT: A potential therapeutic biomarker in patients with glioblastoma correlates with cell proliferation and invasion
}

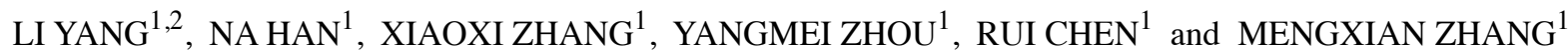 \\ ${ }^{1}$ Department of Oncology, Tongji Hospital, Tongji Medical College, Huazhong University of Science and Technology, \\ Wuhan, Hubei 430030; ${ }^{2}$ Department of Oncology, Minda Hospital, Hubei Minzu University, Enshi, Hubei 445000, P.R. China
}

Received September 9, 2019; Accepted February 26, 2020

DOI: $10.3892 /$ or.2020.7573

\begin{abstract}
Glioblastoma (GBM) is the most aggressive primary intracranial tumor in adults. Chemoradiotherapy resistance and recurrence after surgery are the main malignant progression factors, leading to a high mortality rate. Therefore, the exploration of novel biomarkers and molecular mechanisms of GBM is urgent. Differentially expressed genes (DEGs) of GBM were screened in a TCGA dataset. Homo sapiens ZW10 interacting kinetochore protein (ZWINT) was found to be upregulated in GBM, which was confirmed by immunohistochemical staining of a tissue microarray. Gene Ontology (GO) annotation and Kyoto Encyclopedia of Genes and Genomes (KEGG) pathway enrichment analysis were performed using the Database for Annotation, Visualization and Integrated Discovery (DAVID) database. A protein-protein interaction (PPI) network was established by the STRING database, and hub genes were visualized by Cytoscape. The correlation results were verified with the GSE15824 dataset. Bioinformatic analysis confirmed that ZWINT was significantly positively correlated with kinetochore protein NDC80 homolog (NDC80), serine/threonine-protein kinase PLK1 (PLK1) and spindle and kinetochore associated complex subunit 1 (SKA1) and together are involved in regulating mitosis and the cell cycle of GBM. ZWINT expression was knocked down in U251 and U87 MG GBM cells by lentiviral vectors carrying a small hairpin RNA (shRNA) targeting ZWINT. The effect of ZWINT silencing on cell proliferation, invasion and apoptosis was determined by the Celigo assay, MTT assay, Transwell assay, flow cytometry and caspase-3/7 assay in vitro. A subcutaneous xenograft tumor model was established to explore the influence of ZWINT knockdown on GBM growth in vivo. Our preliminary study demonstrated that ZWINT knockdown
\end{abstract}

Correspondence to: Professor Mengxian Zhang, Department of Oncology, Tongji Hospital, Tongji Medical College, Huazhong University of Science and Technology, 1095 Jiefang Road, Wuhan, Hubei 430030, P.R. China

E-mail: zhangmx73@163.com

Key words: glioblastoma, bioinformatics, TCGA, GEO, ZWINT, shRNA effectively inhibited proliferation and invasion and induced apoptosis of GBM cells and notably suppressed GBM growth in vivo. Therefore, we speculate that ZWINT may be a potential therapeutic biomarker for GBM, with NDC80 and PLK1 conjointly involved in regulating cell division and the mitotic cell cycle.

\section{Introduction}

Glioblastoma (GBM) is the most common primary malignant brain tumor in adults. High-grade GBM remains a devastating disease despite maximal therapy with surgery, radiation and chemotherapy with temozolomide (TMZ). GBM patients present with only a 15 - to 19-month median overall survival rate due to chemoradiotherapy resistance and recurrence (1). Recent studies have identified several molecular alterations involved in GBM carcinogenesis, progression, chemotherapeutic resistance and recurrence, such as IDH1, 1p/19q, MGMT, ATRX and PTEN, but the precise mechanisms underlying this malignancy and its rapid recurrence have not been fully elucidated (2-4). Targeting key genes and signaling pathways is considered a promising approach for the diagnosis and treatment of cancer. Therefore, finding potential biomarkers with bioinformatic analysis contributes to a better understanding of the occurrence and development of GBM.

Homo sapiens $\mathrm{ZW} 10$ interacting kinetochore protein (ZWINT) is a known component of the kinetochore complex required for the mitotic spindle checkpoint and plays crucial roles in mitotic cycle maintenance (5). The kinetochore is a highly complex structure that is central to many essential activities during cell division. The kinetochore, a tri-laminar plate to which microtubules attach, connects chromosomes to the spindle to ensure the accurate segregation of chromosomes in mitosis and meiosis (6). ZWINT encodes a protein that is clearly involved in kinetochore function, possibly by regulating the association between ZW10 and centromere complexes during mitotic and mitotic prometaphase (7). It is known that abnormal mitosis is a common feature of most malignancies. Although the exact role of the molecular makeup of the kinetochore and how individual components of the kinetochore interact with each other are unknown, growing evidence shows that ZWINT is often highly expressed in a number of human cancers and is linked with poor clinical prognosis and early recurrence (8-10). However, its role in human GBM 
remains unclear. In our research, we aimed to investigate the expression of ZWINT and its biological significance in this primary malignancy.

\section{Materials and methods}

Dataset processing. TCGA (The Cancer Genome Atlas, https://cancergenome.nih.gov/) is a public repository for data storage that is freely available to users. A variety of human cancer and tumor subtype genomic mutation profiles (11), transcriptomic data (12), and clinical data (13) have been generated, providing a systematic characterization of methylation (14), miRNA expression (15), and oncogenic processes (16). Gene expression profiles of GBM were downloaded from the TCGA dataset, which contains 529 GBM samples and 10 normal samples. The data of the expression profile chip level 3 of these samples were sorted out for analyzing the differentially expressed genes (DEGs). However, multiple sets of data were assessed for certain samples in practice, thus the actual number of downloaded files was more than the original samples (548 GBM samples vs. 10 normal samples). We used $\mathrm{P}<0.05$ and $\mathrm{IFCl}$ (fold change) $\geq 2$ as the criteria, and the edgeR (https://bioconductor.org/packages/release/bioc/html/edgeR. html) $(17,18)$ package in R 3.4.1 was used to identify DEGs in the GBM samples compared with normal brain samples to finally obtain the DEG list. Another gene dataset, GSE15824 (19), was downloaded from the NCBI GEO database (https://www.ncbi.nlm.nih.gov/geo/), and GPL570 was the platform file. Standardization data were carried out using the RMA algorithm of the Affy (http://bioconductor.org/packages/release/bioc/html/Affy.html) (20) package in R software, which were used for the subsequent analysis.

GO and KEGG pathway analyses. Database for Annotation, Visualization and Integrated Discovery (DAVID) 6.8 (http://david.abcc.ncifcrf.gov/) is an online platform that is used for gene annotation, visualization and integrated discovery $(21,22)$. Gene Ontology (GO) and Kyoto Encyclopedia of Genes and Genomes (KEGG) pathway enrichment analyses were implemented with the DAVID database. Using this comprehensive tool, we can understand the biological meaning behind the DEGs more quickly and effectively. $\mathrm{P}<0.05$ indicated a statistically significant difference.

PPI network. The Search Tool for the Retrieval of Interacting Genes (STRING, http://string-db.org) database was queried to construct the protein-protein interaction (PPI) network (23). A confidence score $\geq 0.9$ was set as the cutoff criterion, and disconnected nodes were excluded from the network. CytoHubba and Molecular Complex Detection (MCODE) in Cytoscape 3.5.1 were performed to identify hub genes and significant modules of the PPI network $(24,25)$. The filter conditions were as follows: Degree cutoff $=2$, node score cutoff $=0.2, \mathrm{k}$-core $=2$, and max. depth=100.

Correlation analysis. The GSE15824 dataset contained 40 GBM samples and 5 normal brain tissues. R software was used to evaluate the correlation between the expression of ZWINT and other related hub genes, and the Pearson correlation coefficient $(\mathrm{R})$ was used to reflect the degree of linear correlations.
The comparison criteria were as follows: Within $-1<R<1$, if $\mathrm{R}>0$, indicated a positive correlation between the two genes; $\mathrm{R}<0$ indicated a negative correlation, and the greater the absolute value of $R$, the more significant was the negative correlation; and $\mathrm{R}=0$ indicated nonlinear correlation.

Cell culture. The human GBM cell lines U251, U87 MG, A172, and U373 were obtained from the American Type Culture Collection (ATCC). The specific origin of U87 MG cells is unknown in the ATCC version. These four cell lines were authenticated using short tandem repeat (STR) profiling analysis following ISO 9001:2008 and ISO/IEC 17025:2005 quality standards. The percent matches for U251, U373, A172 and U87 MG cells were 100, 89, 100 and 100\%, respectively. They were cultured in DMEM supplemented with $10 \%$ fetal bovine serum (FBS) (Ausbian, USA) and incubated at $37^{\circ} \mathrm{C}$ in a humidified atmosphere of $5 \% \mathrm{CO}_{2}$.

Immunohistochemistry microarray. A commercially available GBM tissue microarray (TMA) slide was purchased from Shanghai GeneChem Co., Ltd., for IHC analysis. A specific primary antibody against ZWINT was utilized for immunohistochemistry (IHC) with a 2-step protocol. IHC scores were calculated according to the intensity and proportion of positive staining. Staining intensity was classified as 0 (negative), 1 (weak), 2 (moderate) and 3 (strong). The positive cell ratio of the stained cells was scored as $0(<1 \%), 1(1-25 \%), 2$ (26-50\%), $3(51-75 \%)$ and $4(76-100 \%)$. The multiplied result of the two scores represented the protein levels of ZWINT, and an immunoreactive score (IRS) $>6$ was defined as a high expression level of ZWINT, while an IRS $\leq 6$ indicated a low level of ZWINT.

Quantitative PCR ( $q P C R)$. Total RNA was extracted from cells using a TRIzol kit (cat. no. 3101-100, Pufei Biotechnology), and complementary DNA (cDNA) was synthesized with the M-MLV reagent (Promega). The SYBR Master Mix (Takara) and Mx3000P Real-Time PCR machine were used to perform RT-qPCR. The PCR primers (GeneChem) were ZWINT-F, 5'-CACGTAGAGGCCATCAAAATTGG-3' and ZWINT-R, 5'-CGGAGTTGTGTCCGTTTCCT-3'; GAPDH-F, 5'-TGA CTTCAACAGCGACACCCA-3' and GAPDH-R, 5'-CAC CCTGTTGCTGTAGCCAAA-3'. Relative quantification was calculated using the $\Delta \Delta \mathrm{Cq}$ method (26), normalized based on $\mathrm{GAPDH}$, and conducted in triplicate.

ZWINT shRNA design and vector transfection. According to the cDNA sequence of ZWINT (Gene Bank accession no. NM_007057) and the design principle of the small hairpin RNA (shRNA), three target characteristic sequences and nonspecific sequences were selected. shRNA against ZWINT was transfected into $293 \mathrm{~T}$ cells using Lipofectamine $2000^{\mathrm{TM}}$ reagent (Invitrogen; Thermo Fisher Scientific, Inc.), and the expression of ZWINT at the protein level was detected by Western blot analylsis to determine the transfection efficiency. The optimal target sequence was selected: 5'-TTCTCCGAA CGTGTCACGT-3'. ZWINT shRNA and the control shRNA were provided by GeneChem Co., Ltd. ZWINT shRNA was used to knock down ZWINT expression in the U251 and U87 MG cells with Lipofectamine 2000 reagent. At 72 h after 
Table I. Functional and pathway enrichment analysis of DEGs in GBM.

\begin{tabular}{|c|c|c|c|c|}
\hline Category & GO/KEGG ID & Description & Count & FDR \\
\hline \multicolumn{5}{|c|}{ Upregulated } \\
\hline \multirow[t]{5}{*}{$\mathrm{BP}$} & GO:0007067 & Mitotic nuclear division & 64 & $3.18 \mathrm{E}-15$ \\
\hline & GO:0051301 & Cell division & 78 & $5.45 \mathrm{R}-15$ \\
\hline & GO:0007062 & Sister chromatid cohesion & 34 & $5.28 \mathrm{E}-10$ \\
\hline & GO:0008283 & Cell proliferation & 58 & $1.90 \mathrm{E}-04$ \\
\hline & GO:0000086 & $\mathrm{G} 2 / \mathrm{M}$ transition of mitotic cell cycle & 27 & 0.018169 \\
\hline \multirow[t]{5}{*}{$\mathrm{CC}$} & GO:0015935 & Small ribosomal subunit & 20 & $3.22 \mathrm{E}-13$ \\
\hline & GO:0005829 & Cytosol & 343 & $9.55 \mathrm{E}-10$ \\
\hline & GO:0005634 & Nucleus & 497 & 4.31E-07 \\
\hline & GO:0005737 & Cytoplasm & 478 & 0.018475 \\
\hline & GO:0000777 & Condensed chromosome kinetochore & 20 & 0.018475 \\
\hline \multirow[t]{10}{*}{$\mathrm{MF}$} & GO:0005515 & Protein binding & 745 & 4.65E-06 \\
\hline & GO:0001077 & Transcriptional activator activity & 42 & $3.17 \mathrm{E}-04$ \\
\hline & GO:0000978 & $\begin{array}{l}\text { RNA polymerase II core promoter proximal region } \\
\text { sequence-specific DNA binding }\end{array}$ & 54 & $9.19 \mathrm{E}-04$ \\
\hline & GO:0003682 & Chromatin binding & 56 & 0.003782 \\
\hline & GO:0043565 & Sequence-specific DNA binding & 68 & 0.006257 \\
\hline & KEGG:hsa04014 & MAPK signaling pathway & 84 & $1.02 \mathrm{E}-05$ \\
\hline & KEGG:hsa04022 & cGMP-PKG signaling pathway & 55 & 0.000239 \\
\hline & KEGG:hsa04110 & Cell cycle & 44 & 0.000364 \\
\hline & KEGG:hsa04014 & Ras signaling pathway & 66 & 0.012203 \\
\hline & KEGG:hsa03030 & DNA replication & 14 & 0.036848 \\
\hline \multicolumn{5}{|c|}{ Downregulated } \\
\hline \multirow[t]{5}{*}{$\mathrm{BP}$} & GO:2000463 & Positive regulation of excitatory postsynaptic potential & 14 & $8.31 \mathrm{E}-05$ \\
\hline & GO:0006836 & Neurotransmitter transport & 15 & $6.88 \mathrm{E}-04$ \\
\hline & GO:0007214 & $\gamma$-aminobutyric acid signaling pathway & 12 & 0.045329 \\
\hline & GO:0007193 & $\begin{array}{l}\text { Adenylate cyclase-inhibiting G-protein coupled } \\
\text { receptor signaling pathway }\end{array}$ & 18 & 0.039186 \\
\hline & GO:0007264 & Small GTPase mediated signal transduction & 54 & 0.045329 \\
\hline \multirow[t]{5}{*}{$\mathrm{CC}$} & GO:0030426 & Growth cone & 39 & $1.00 \mathrm{E}-05$ \\
\hline & GO:0043679 & Axon terminus & 22 & $8.72 \mathrm{E}-05$ \\
\hline & GO:0032809 & Neuronal cell body membrane & 13 & $1.61 \mathrm{E}-04$ \\
\hline & GO:0048471 & Perinuclear region of cytoplasm & 118 & 0.002053 \\
\hline & GO:0098793 & Presynapse & 23 & 0.006006 \\
\hline \multirow[t]{5}{*}{ MF } & GO:0005088 & Ras guanyl-nucleotide exchange factor activity & 34 & 0.001815 \\
\hline & GO:0019905 & Syntaxin binding & 26 & 0.003059 \\
\hline & GO:0044325 & Ion channel binding & 32 & 0.010635 \\
\hline & GO:0004683 & Calmodulin-dependent protein kinase activity & 12 & 0.023968 \\
\hline & GO:0004674 & Protein serine/threonine kinase activity & 74 & 0.032426 \\
\hline
\end{tabular}

To note, according to the P-value in our study, five terms were selected for each category. DEGs, differentially expressed genes; GBM, glioblastoma; FDR, false discovery rate; GO, Gene Ontology; KEGG, Kyoto Encyclopedia of Genes and Genomes; BP, biological process; $\mathrm{CC}$, cellular component; MF, molecular function.

transfection, U251 and U87 cells were harvested for later research.

Western blot assay. 293T cells were lysed in radioimmunoprecipitation assay (RIPA) buffer after transfection with shRNA for 72 h. A BCA Protein Assay Kit was used to detect the total protein concentration. Equal amounts of protein lysates were electrophoresed in 10\% SDS-PAGE gels and then transferred to polyvinylidene difluoride (PVDF) membranes, which were blocked by Tris-buffered saline with Tween (TBST) containing 5\% milk. Protein expression was probed with mouse anti-FLAG (F1804; Sigma-Aldrich; Merck KGaA) and goat anti-mouse IgG (SC-2005; Santa Cruz Biotechnology, Inc.). Protein expression in 293T cells that were exogenously 
A

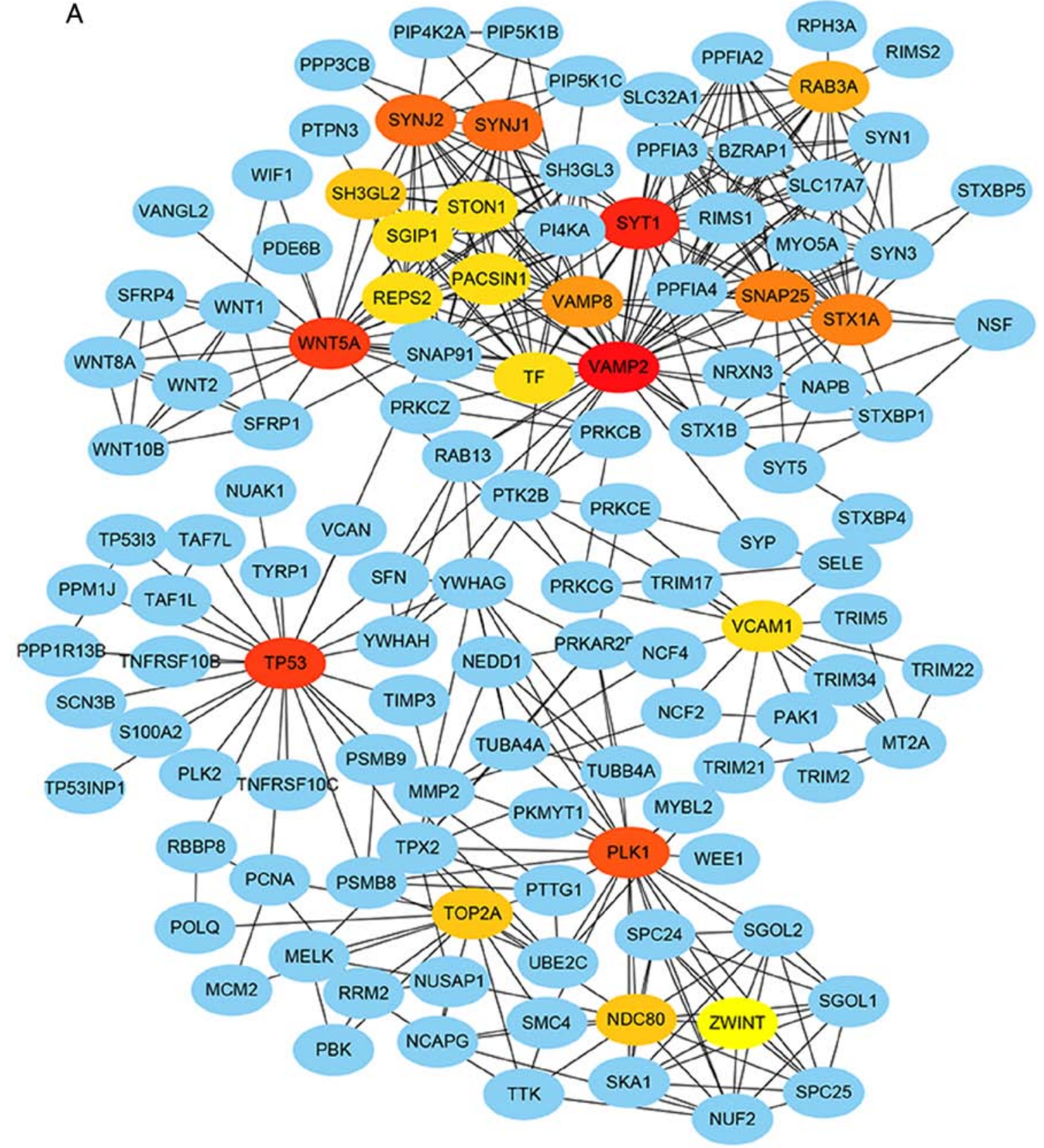

B

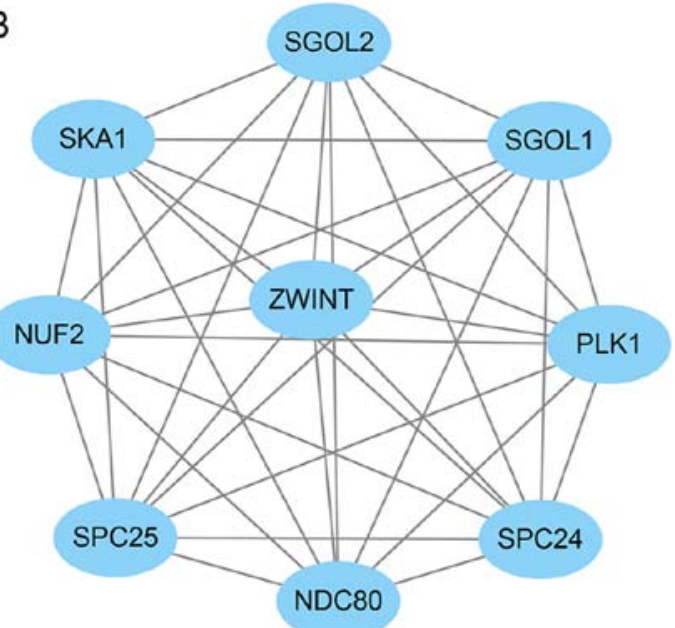

Figure 1. Hub genes and a significant module in the PPI network. (A) Hub genes. The ovals represent the DEGs, and the lines show the interactions between them. The red and yellow nodes represent the rank of hub genes from high to low according to the degree. (B) A significant module. The significant module contains DEGs that may play an important role in the PPI network. PPI, protein-protein interaction; DEG, differentially expressed genes. 

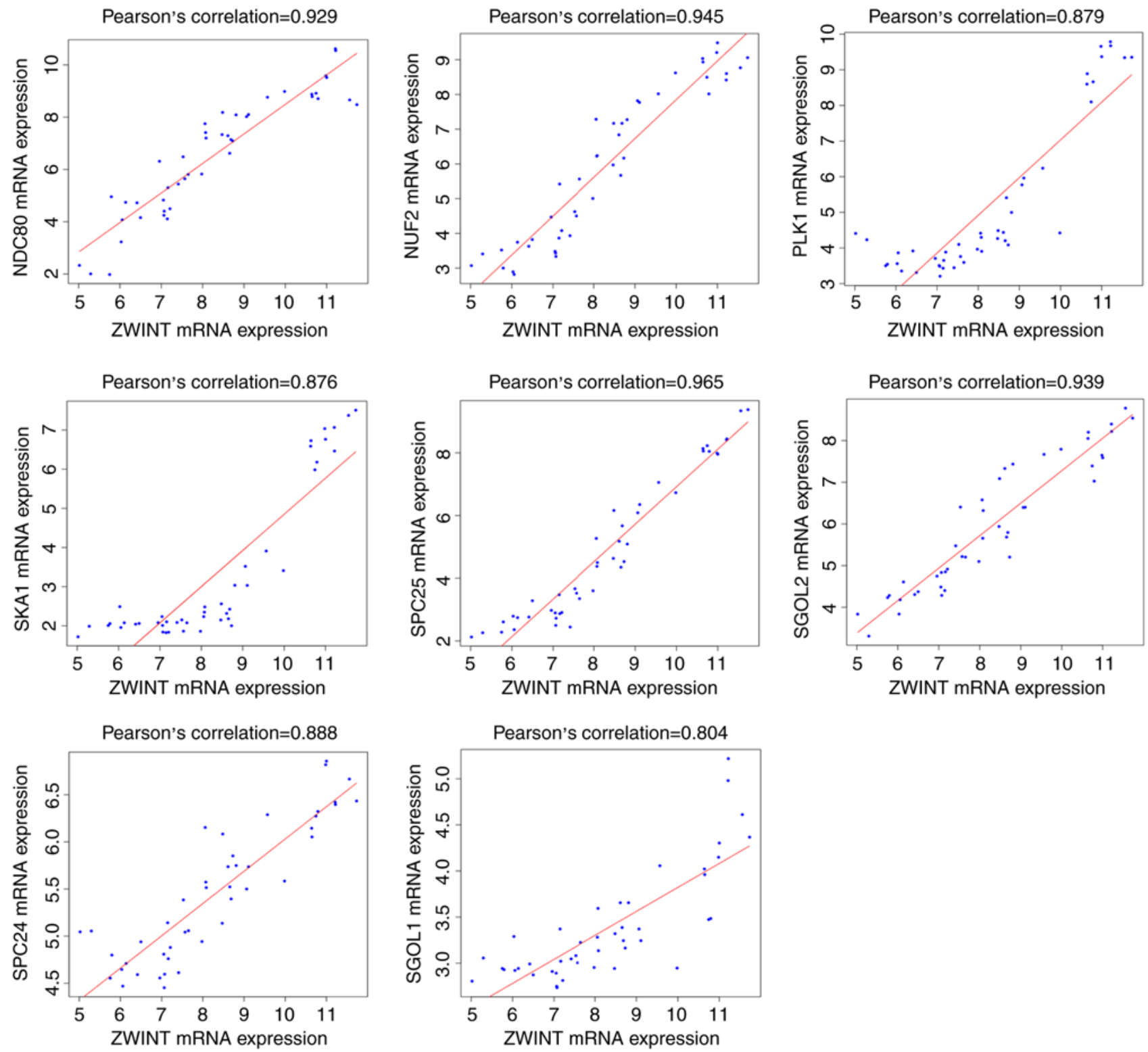

Figure 2. Correlation between the expression of ZWINT and the levels of other hub genes in GBM tissues. Correlation analysis of the public dataset GSE15824 showed that ZWINT mRNA expression was significantly positively correlated with the cell division and mitotic cell cycle markers $N D C 80, P L K 1, N U F 2$, SKA1, SPC24, SPC25, SGOL1 and SGOL2. ZWINT, Homo sapiens ZW10 interacting kinetochore protein; GBM, glioblastoma; NDC80, NDC80 homolog; $P L K 1$, serine/threonine-protein kinase PLK1; NUF2, NUF2 component of NDC80 kinetochore complex; SKA1, spindle and kinetochore associated complex subunit 1; SPC24, SPC24 component of NDC80 kinetochore complex; SPC25, SPC25 component of NDC80 kinetochore complex; SGOL1, shugoshin 1; SGOL2, shugoshin 2.

transfected with a plasmid encoding FLAG-tagged ZWINT was detected by mouse anti-FLAG and mouse anti-GAPDH (SC-32233; Santa Cruz Biotechnology, Inc.). The ECL-PLUS kit (Thermo Fisher Scientific. Inc) was used to detect the signals on X-ray film. GAPDH was used as the reference protein to calculate the relative protein levels. Independent tests were performed in triplicate.

Cell growth and MTT proliferation assay. The number of cells at each time point was counted by a Celigo Image Cytometer (Nexcelom Bioscience). Briefly, the GBM cells were cultured in 96-well plates at an initial density of $2 \times 10^{3}$ cells/well. Each group had three wells $(10 \mu \mathrm{l} /$ well $)$ and was incubated at $37^{\circ} \mathrm{C}$ in an atmosphere of $5 \% \mathrm{CO}_{2}$. Fluorescence photomicrographs were captured, and cells with green fluorescence were measured by a Celigo Image Cytometer. Cell growth curves were generated for a time course of 5 days. MTT [3(4,5-dimethylthiazol-2-yl)-2,5-diphenyltetrazolium bromide] (Genview Scientific. Inc.) was used to measure cell viability. The infected cells $\left(2 \times 10^{3}\right.$ cells/well $)$ were collected and reseeded in 96-well plates. From the first to the 5th day, a total of $20 \mu \mathrm{l}$ MTT solution $(5 \mathrm{mg} / \mathrm{ml})$ was added to the cells. Subsequently, the supernatants were removed, $100 \mu \mathrm{l}$ DMSO was added to each well, and the plates were oscillated for $3 \mathrm{~min}$. Finally, the optical density (OD) at $490 \mathrm{~nm}$ was measured with a microplate reader.

Transwell chamber assay. U251 and U87 MG cells were transfected with ZWINT shRNA or NC, cultured for $72 \mathrm{~h}$ and harvested. The invasion assay, in which the Transwell 
A

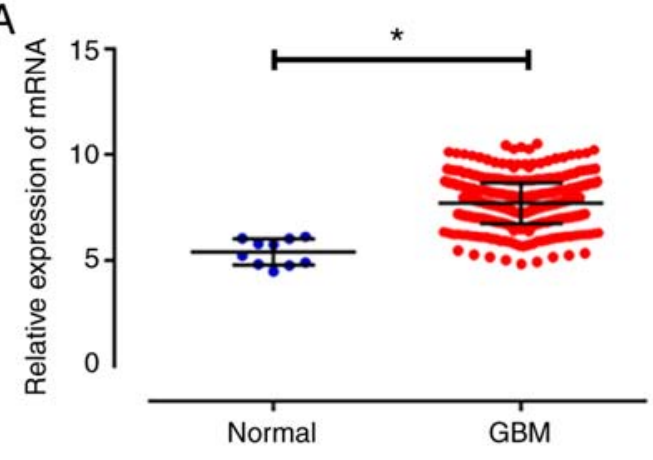

B

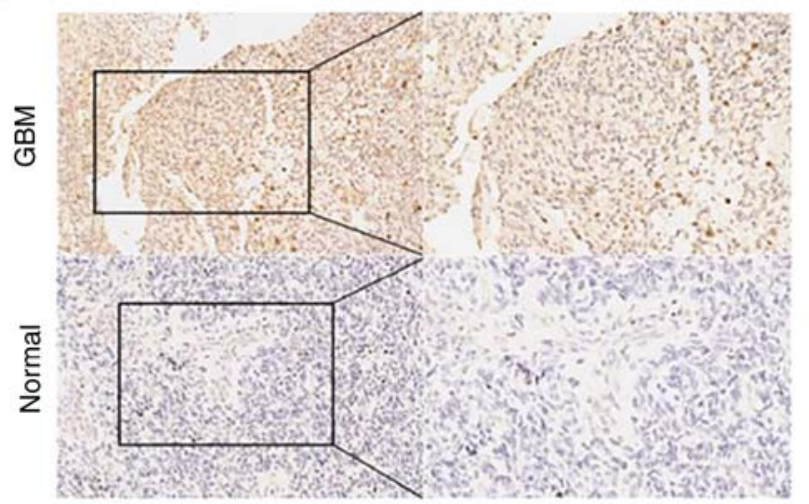

C

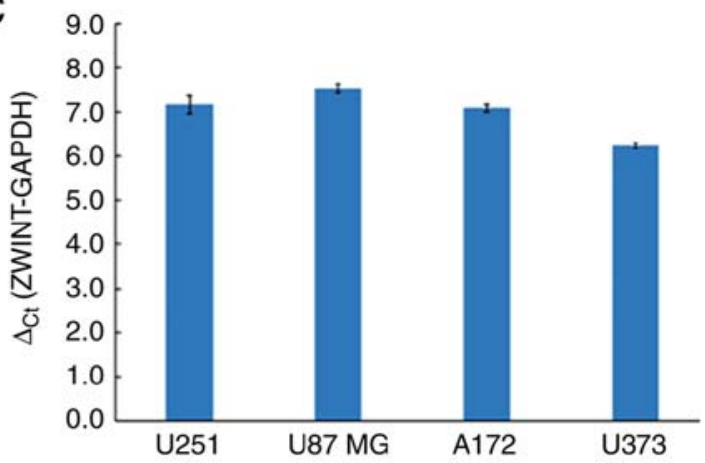

D

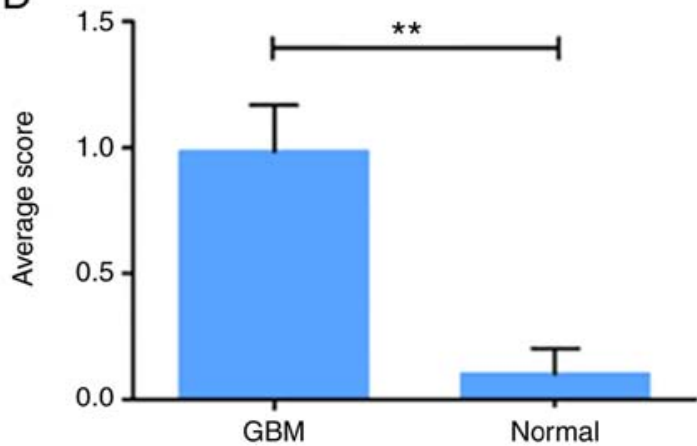

Figure 3. Expression level of ZWINT is upregulated in GBM tissues and cell lines. (A) The mRNA level of ZWINT was significantly higher in GBM samples than that noted in the normal samples based on the TCGA dataset (548 GBM samples vs. 10 normal samples. " $\mathrm{P}<0.05$, t-test). (B) Representative immunohistochemistry (IHC) images of ZWINT expression in GBM tissue microarrays. Positive ZWINT staining was found in cytoplasmic tumor cells compared with normal brain tissues. (C) qPCR was used to determine $Z W I N T$ mRNA expression levels in glioma cell lines $(\Delta \mathrm{Ct} \leq 12$ indicates a significantly higher mRNA expression level of ZWINT in GBM cells). (D) Scoring results showed that ZWINT was overexpressed in GBM tissues, and the difference was statistically significant $\left({ }^{* *} \mathrm{P}<0.01\right)$. ZWINT, Homo sapiens ZW10 interacting kinetochore protein; GBM, glioblastoma.

chamber was coated with Matrigel, was conducted according to the manufacturer's instructions for the Corning Invasion Kit (Corning Inc.). A total of $500 \mu \mathrm{l}$ cell suspension in FBS-free DMEM ( $10^{5}$ cells/well in a 24 -well plate) was added to the upper chamber, and $750 \mu \mathrm{l}$ DMEM containing 30\% FBS was added to the lower chamber. Cells were removed from the upper chamber of the filter with a cotton swab after $24 \mathrm{~h}$ of incubation at $37^{\circ} \mathrm{C}$. Cells on the underside were washed with PBS, stained with Giemsa, captured with a digital camera and counted in 5 randomly selected fields of vision at x100 magnification and 9 randomly fields of vision at x200 magnification under a phase contrast microscope.

Flow cytometry and caspase-3/7 assay. Apoptotic cells were assessed using the Annexin V apoptosis kit (88-8007; eBioscience). In short, cells were trypsinized, washed and centrifuged at $283 \mathrm{x} \mathrm{g}$ for $5 \mathrm{~min}$. Then, $1 \mathrm{X}$ binding buffer was used to wash the cells again by resuspending the cells in $200 \mu \mathrm{l}$. Then, the cells were incubated with $10 \mu \mathrm{l}$ Annexin V-APC at room temperature for $15 \mathrm{~min}$ in the dark. Finally, the Annexin V-stained cells were analyzed with a flow cytometer (Millipore) to determine the proportion of apoptotic cells.

Caspase-3/7 are central effector caspases in apoptosis and are usually used to measure apoptotic activities. GBM cells were first transfected with the ZWINT shRNA and NC. Next, $1 \times 10^{4}$ infected cells/well were seeded in a 96-well plate. Caspase-Glo 3/7 reagent (100 $\mu 1$, G8091; Promega) was added to each well, the plate was shaken for 30 min constantly and incubation was carried out at ambient temperature for $2 \mathrm{~h}$. The luminescence signal was detected with an M2009PR (Tecan Infinite) plate reader.

Nude mouse study. Animal experiments were approved by the Animal Care Committee of Tongji Hospital of Huazhong University of Science and Technology (Wuhan, Hubei, China), and strictly followed the institutional regulations and state guidelines on experimental animals. BALB/c athymic female nude mice (4-week-old; $20 \mathrm{~g}$ ) were purchased from Shanghai Lingchang Biological Polytron Technologies Inc. They were maintained in a constant temperature, humidity, sterile environment and fed with food and water according to the national regulations. Subcutaneous xenografts of human GBM were

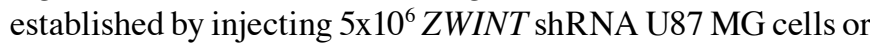
$\mathrm{NC}$ cells into the right hind limbs of the mice; 10 mice in each group. A digital caliper was used to measure tumor growth once every 3 days. Thirty-six days after cell inoculation, the animals were euthanized using an intraperitoneal injection of $150 \mathrm{mg} / \mathrm{kg}$ pentobarbital sodium before collecting the tumors. Tumor tissues were excised and weighted. The tumor volume was defined as $\mathrm{V} \approx \pi / 6 \times \mathrm{L} \times \mathrm{W}^{2}$, where $\mathrm{L}$ stands for the tumor length and $\mathrm{W}$ is the tumor width.

Statistical analysis. SPSS version 20.0 (IBM Corp.) was used for data analysis. Measurement data are expressed as 

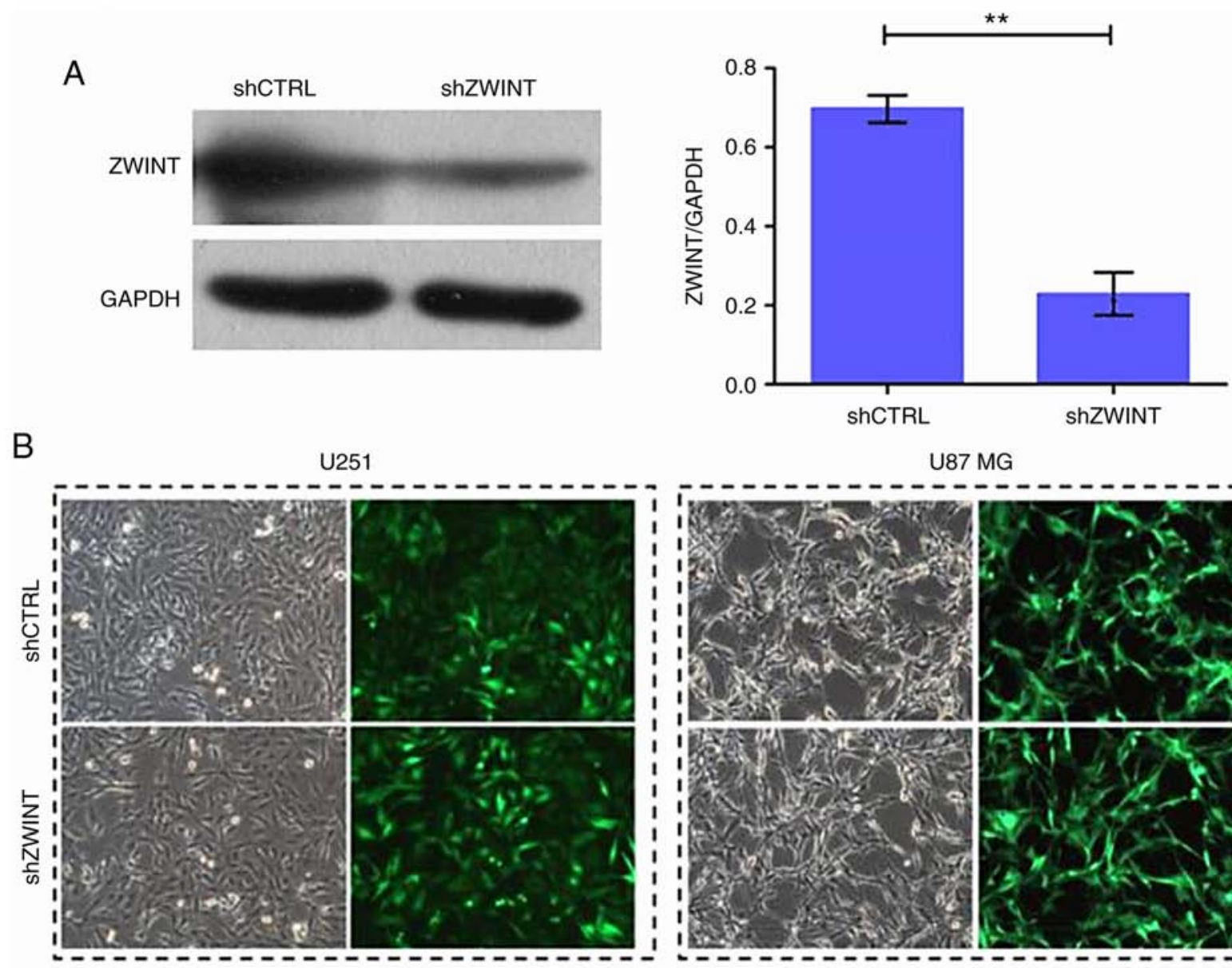

B
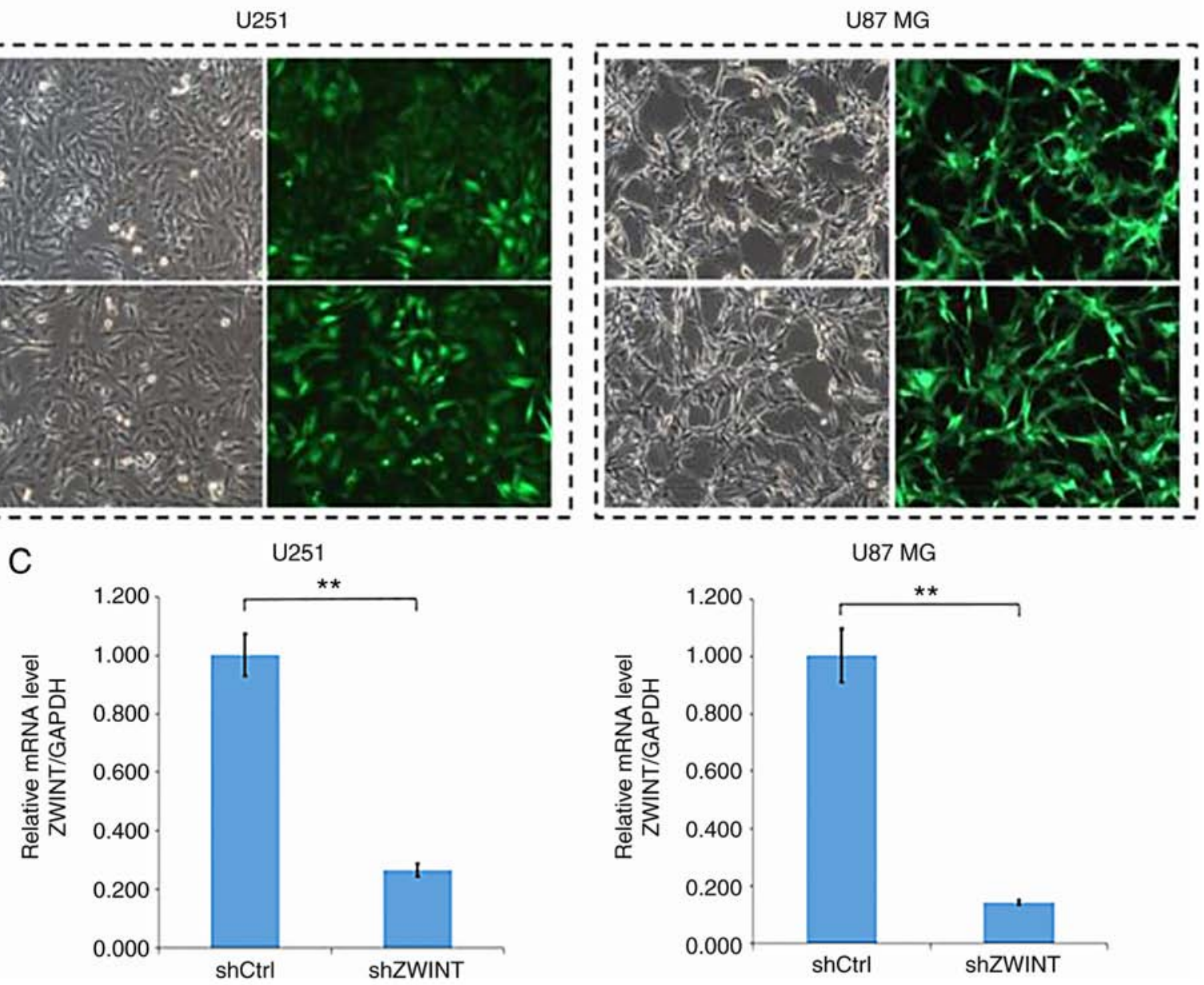

Figure 4. ZWINT expression is effectively knocked down by shRNA. (A) Western blot bands reflect the efficiency of ZWINT silencing in 293T cells. (B) U251 and U87 MG cells were observed by light microscopy and fluorescence microscopy 3 days after infection. The green fluorescence represents infection efficiency, and over $80 \%$ of cells expressed GFP. (C) Confirmation of ZWINT knockdown in U251 and U87 MG cells by qPCR ("* $\mathrm{P}<0.01)$. ZWINT, Homo sapiens ZW10 interacting kinetochore protein.

the mean \pm standard deviation. Student's t-test was used to compare data between two groups. The Pearson correlation coefficient was used for the correlation analysis. $\mathrm{P}<0.05$ was considered to indicate a statistically significant difference.

\section{Results}

GO annotation and KEGG pathway enrichment analysis. The downloaded gene expression profiles in the TCGA database included 2,387 upregulated genes and 3,873 downregulated genes. The GO functional analysis demonstrated that the upregulated genes were mainly involved in biological processes associated with mitotic nuclear division, cell division, sister chromatid cohesion, protein binding and cell proliferation, and the ZWINT gene was mainly enriched in cell division, mitotic sister chromatid segregation, kinetochore, and mitotic cell cycle checkpoint. The downregulated genes were mainly enriched in positive regulation of excitatory postsynaptic potential, neurotransmitter transport, axon terminus, and ion channel binding. Moreover, five significant signaling pathways were overexpressed in the upregulated genes, including the cell cycle, cellular senescence, DNA replication, Ras and MAPK 
A

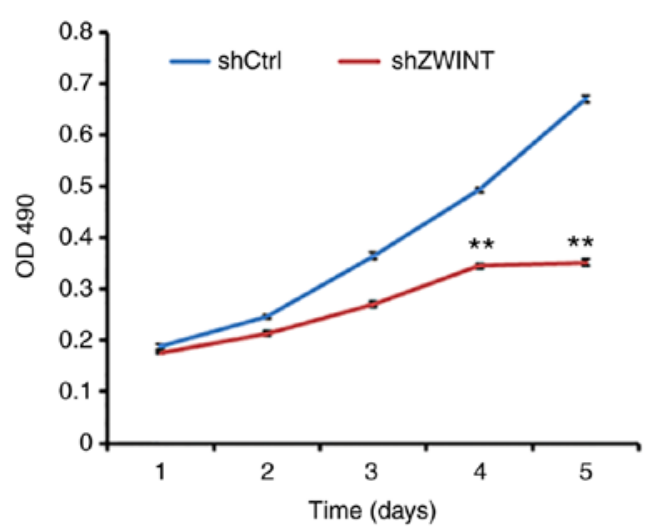

U251

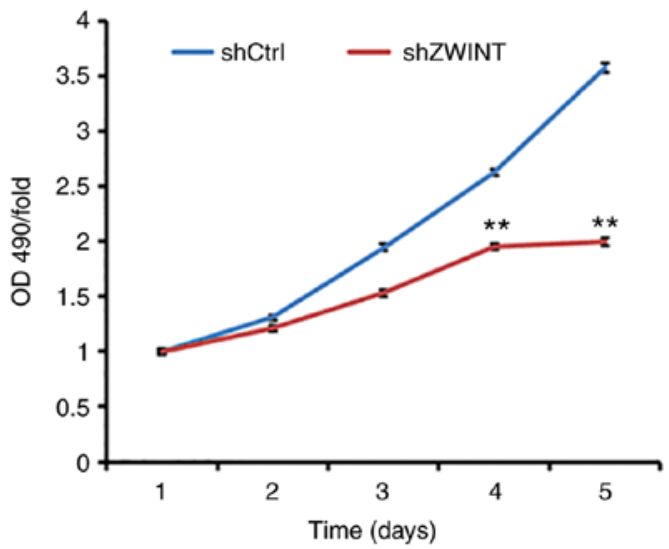

U87 MG
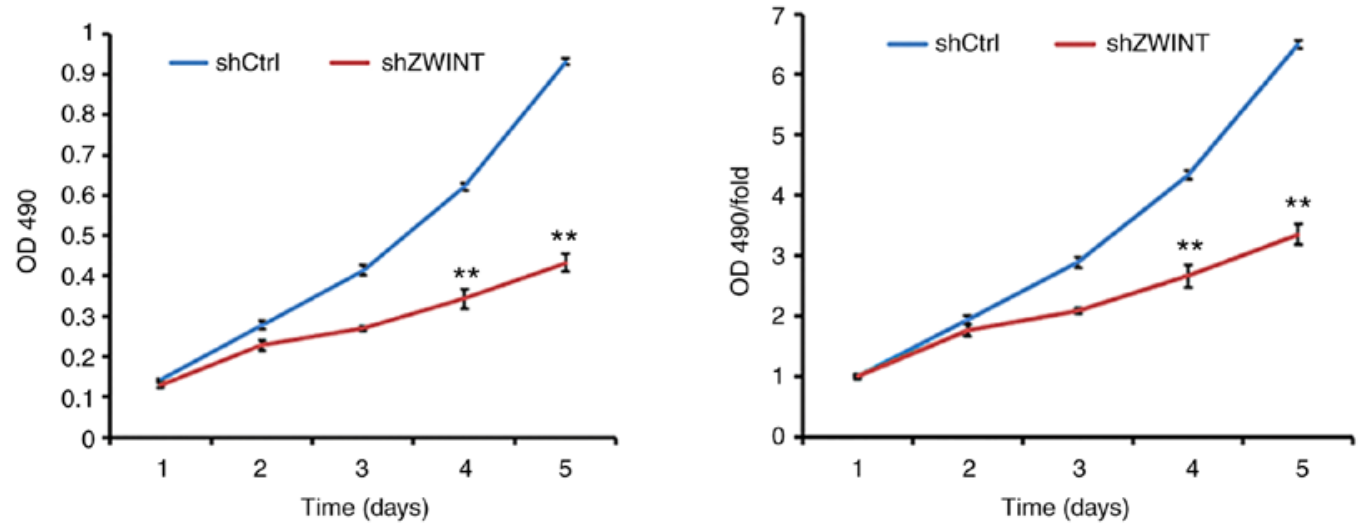

B

U251
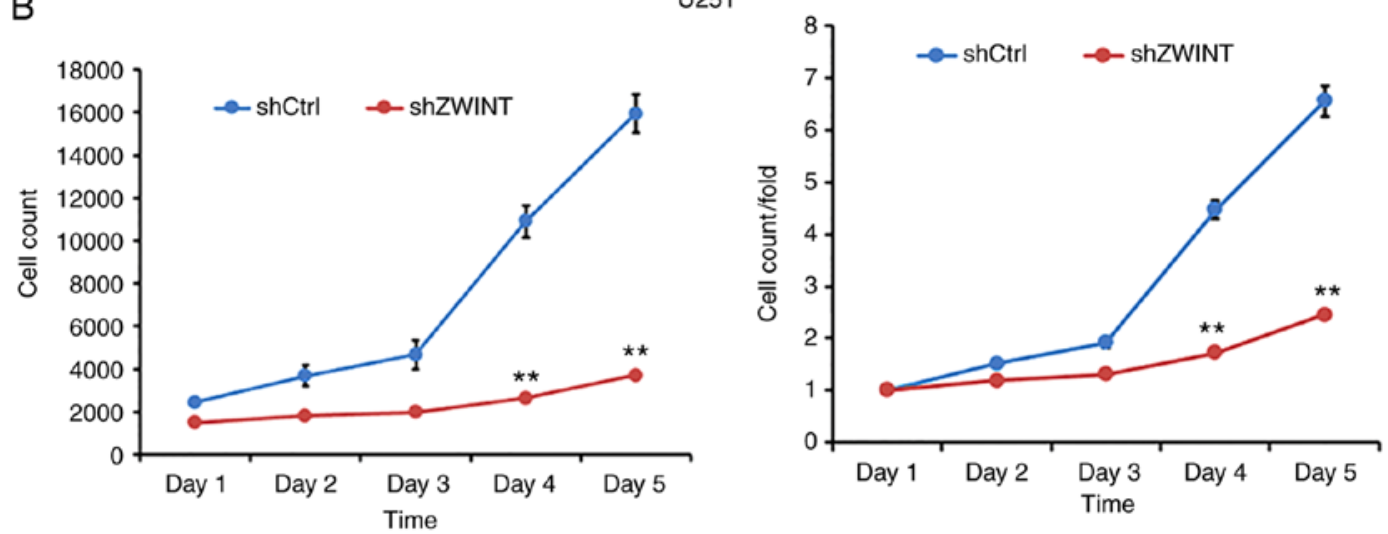

U87 MG
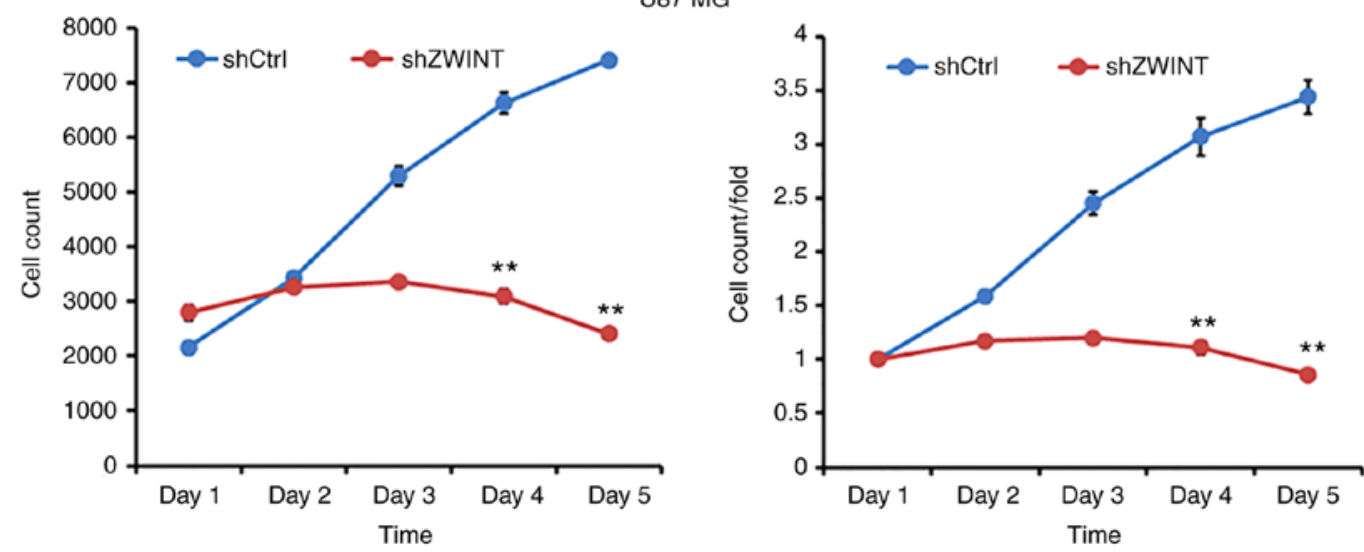

Figure 5. Effects of ZWINT knockdown on tumor cell proliferation. (A) Cell growth curves were plotted based on MTT absorbance. (B) Fluorescent photomicrographs were captured by measuring the cells with green fluorescence in each time series of cell growth, and then cell growth was graphed every day for 5 days by algorithms of the raw image data, as determined by a Celigo asssy ( ${ }^{* *} \mathrm{P}<0.01$, shZWINT vs. shCtrl). ZWINT, Homo sapiens ZW10 interacting kinetochore protein. 
A

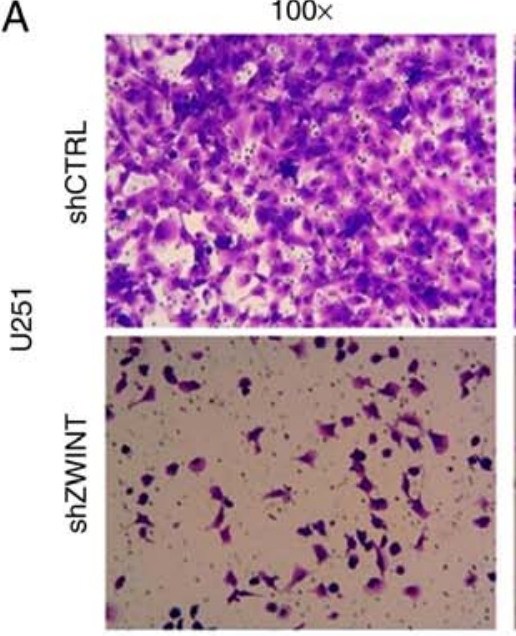

B

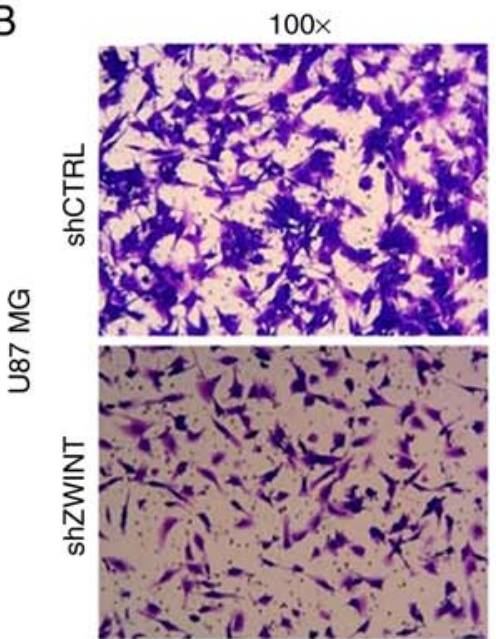

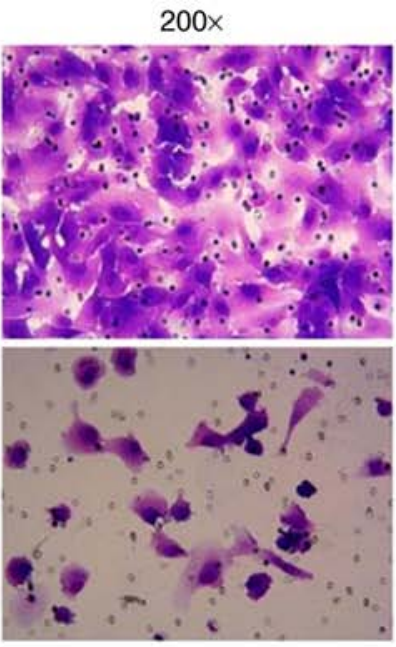

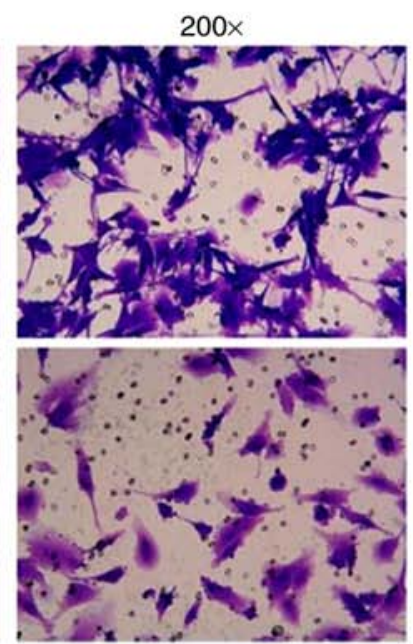

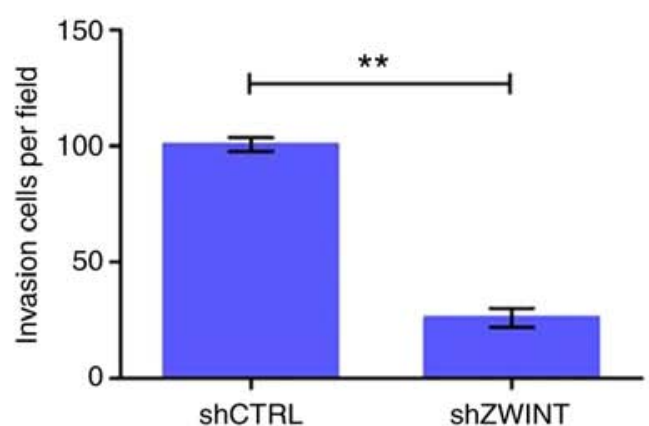

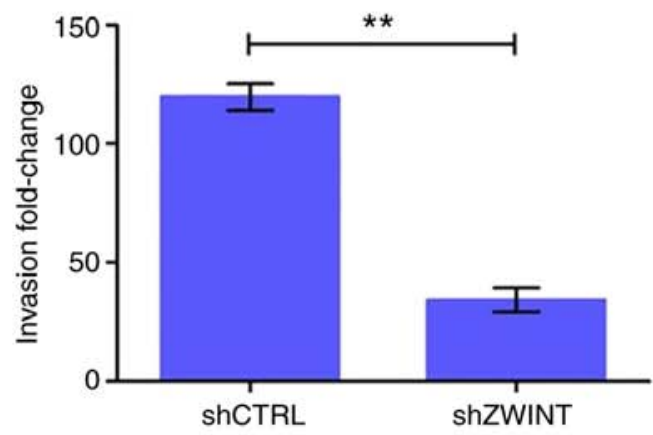

Figure 6. Effects of ZWINT knockdown on tumor cell invasion in U251 (A) and U87 MG (B) cells. Transwell assays showed that knockdown of ZWINT with shRNA (shZWINT) resulted in a significantly lower number of invading cells that migrated through the Matrigel-coated chambers compared to that of the vector control cells (shCtrl) $\left({ }^{* *} \mathrm{P}<0.01\right)$. ZWINT, Homo sapiens ZW10 interacting kinetochore protein.

signaling pathways. Only one KEGG pathway was found for downregulated genes when $\mathrm{P}=0.05$ (Table I).

PPI network. The PPI network of DEGs included 1,843 nodes and 5,116 edges, with each node representing a DEG and the edges indicating the interactions between DEGs. Twenty genes were selected as hub genes, which included tumor protein $\mathrm{p} 53$ (TP53), polo like kinase 1 ( $P L K 1)$, nuclear division cycle 80 (NDC80) and Wnt family member 5A (WNT5A). Moreover, ZWINT interacted with kinetochore protein NDC80 homolog (NDC80), serine/threonine-protein kinase PLK1 (PLK1), spindle and kinetochore associated complex subunit 1 (SKAl) and tripartite motif containing 17 (Terf/TRIM17) (Fig. 1A). The significant module included 9 nodes and 36 edges (Fig. 1B). GO and KEGG pathway enrichment analyses revealed that genes in this module, such as $N D C 80, S K A 1$ and NUF2 component of NDC80 kinetochore complex (NUF2), were mainly associated with cell division, mitotic cell cycle, chromosome segregation, and small GTPase-mediated signal transduction, and they interacted with ZWINT.

Correlation between the expression of ZWINT and other hub genes. Correlation analysis of the public dataset GSE15824 showed that ZWINT mRNA expression was significantly positively correlated with NDC80, PLK1, NUF2, SKA1, SPC24 component of NDC80 kinetochore complex (SPC24), SPC25 component of NDC80 kinetochore complex (SPC25), shugoshin 1 (SGOL1) and shugoshin 2 (SGOL2) expression, and the correlation coefficients were $\mathrm{R}=0.929, \mathrm{R}=0.879$, $\mathrm{R}=0.945, \mathrm{R}=0.876, \mathrm{R}=0.888, \mathrm{R}=0.965, \mathrm{R}=0.804$, and $\mathrm{R}=0.939$, respectively. According to the biological functions of ZWINT, which was enriched in GBM, these 8 hub genes may also be correlated with cell division and mitotic cell cycle (Fig. 2).

High expression of ZWINT in human GBM tissues and cell lines. Our data confirmed that ZWINT was among the upregulated genes, and the expression level of ZWINT mRNA in GBM tissues was significantly higher than that in normal brain tissues (548 GBM samples vs. 10 normal samples, Fig. 3A). In addition, IHC analysis of the TMA was performed to examine the protein level of ZWINT, and a significant increase was identified in GBM tissues compared with the level noted in the paired normal brain tissues (Fig. 3B). Scoring results showed that ZWINT was overexpressed in GBM tissues, and the difference was statistically 
A
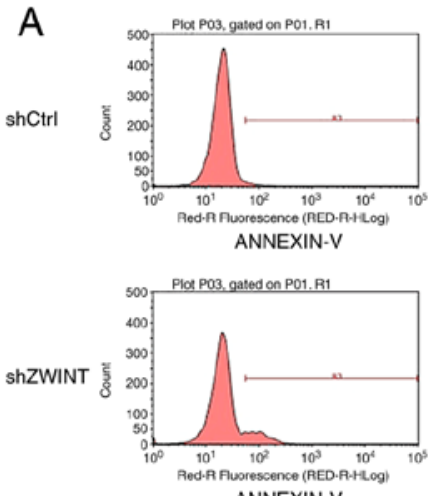

ANNEXIN-V
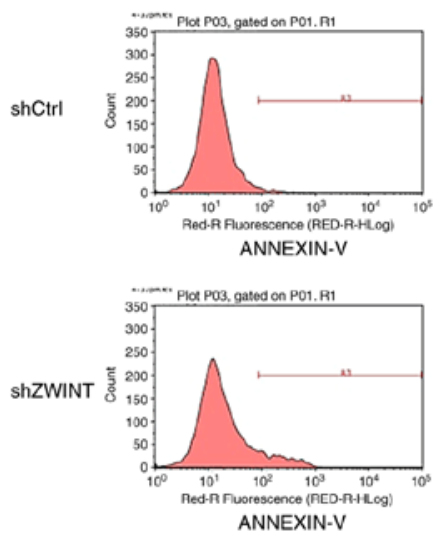

ANNEXIN-V
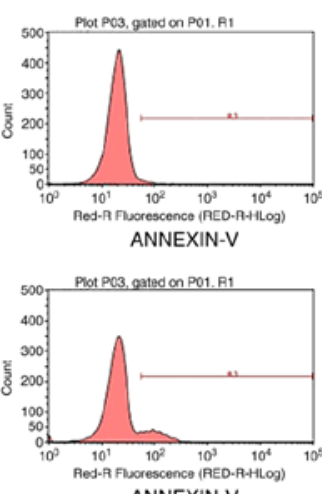

ANNEXIN-V
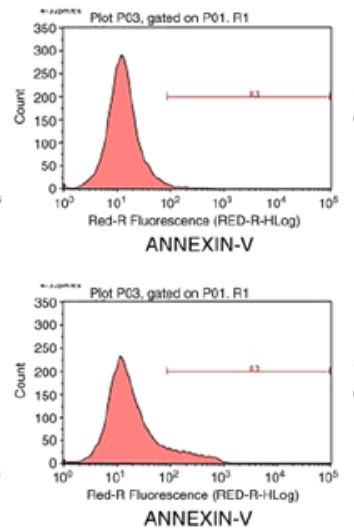

ANNEXIN-V

\section{B}

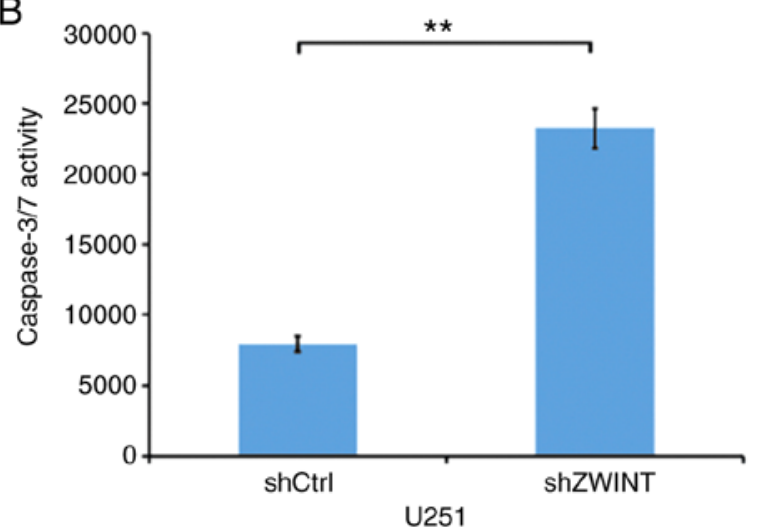

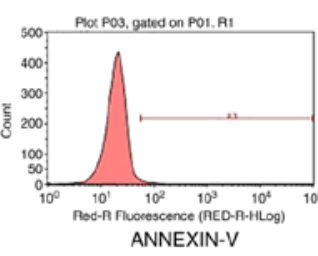
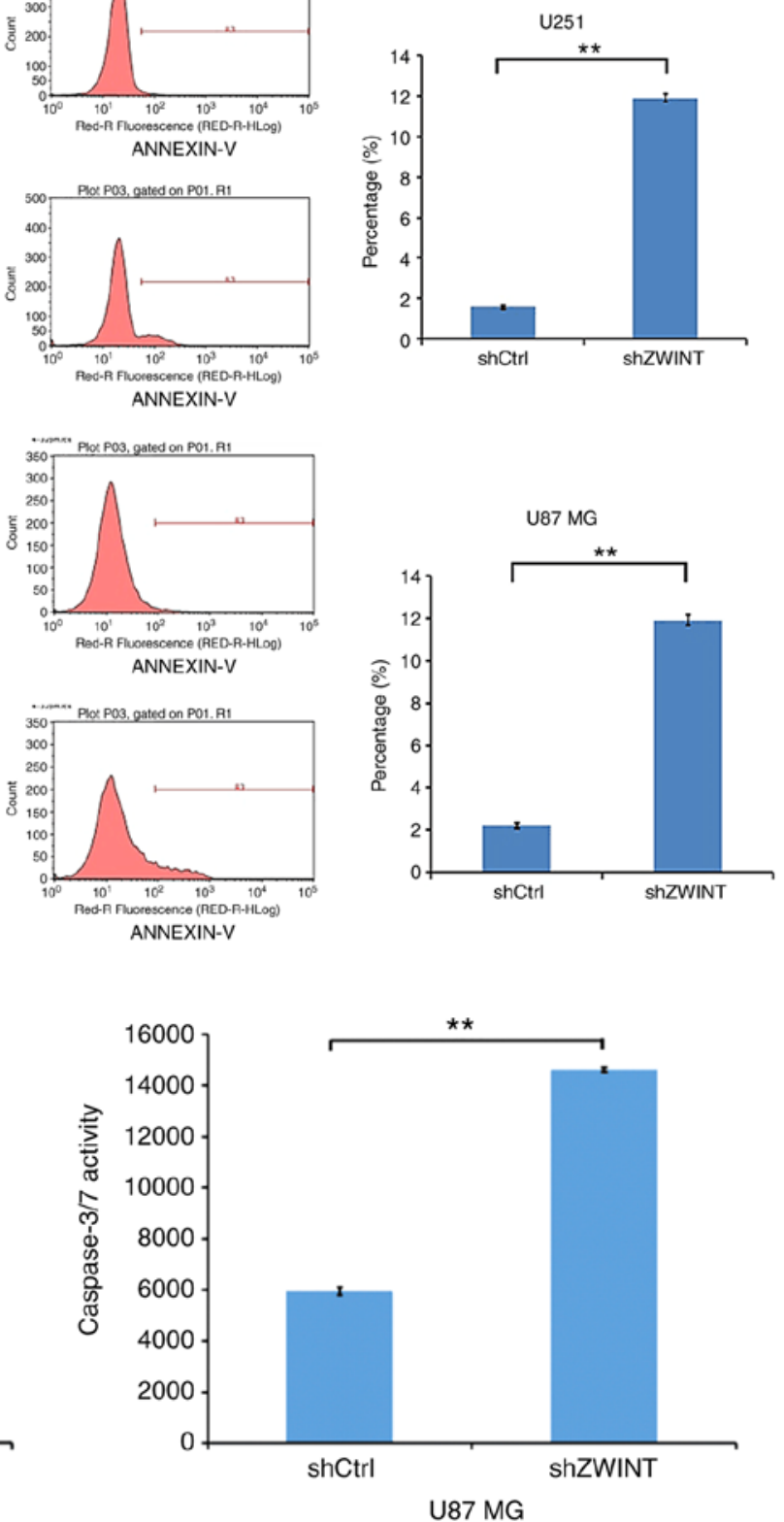

Figure 7. Effects of ZWINT knockdown on tumor cell apoptosis in the U251 and U87 MG cell lines. (A) Cell death was measured by Annexin V-APC staining and flow cytometry. A higher percentage of apoptotic cells was noted in the shZWINT group vs. the shCtrl group. (B) Apoptotic cells were quantified by measuring caspase-3/7 activity $\left({ }^{* *} \mathrm{P}<0.01\right)$. ZWINT, Homo sapiens ZW10 interacting kinetochore protein.

significant $(\mathrm{P}<0.05)$ (Fig. 3D). qPCR analysis revealed that ZWINT was upregulated in all four human GBM cell lines (Fig. 3C). Therefore, U251 and U87 MG cells were selected for ZWINT-knockdown experiments. Western blot analysis revealed that in the $293 \mathrm{~T}$ cell line transfected with shRNA targeting ZWINT (shZWINT), the expression level of ZWINT protein was significantly reduced, indicating an effective lentivirus-delivered shRNA sequence (Fig. 4A). Over $80 \%$ of U251 and U87 MG cells showed green fluorescent protein expression under a fluorescence microscope after infection with the recombinant lentiviruses (Fig. 4B). As detected by $\mathrm{qPCR}$, a significant reduction in the mRNA level was found in U251 and U87 MG cells infected with shZWINT compared with cells infected with shCtrl (Fig. 4C).
Knockdown of ZWINT inhibits GBM cell proliferation. The proliferation activity in U251 and U87 MG ZWINT-knockdown cells was explored by MTT assays. The results showed that the growth rate of shZWINT U251 and U87 MG cells was slower than that of the shCtrl group ( $\mathrm{P}<0.01$, Fig. 5A). Similarly, the growth curve counted by the Celigo Image Cytometer revealed that clonogenic survival in the shZWINT group was markedly decreased (Fig. 5B). Therefore, we speculated that ZWINT may act as an oncogene to promote GBM cell proliferation.

Knockdown of ZWINT suppresses GBM cell invasion. Transwell assays were performed to investigate the role of ZWINT in GBM cell invasion. As expected, the number of cells in the shZWINT group that passed through the 
A

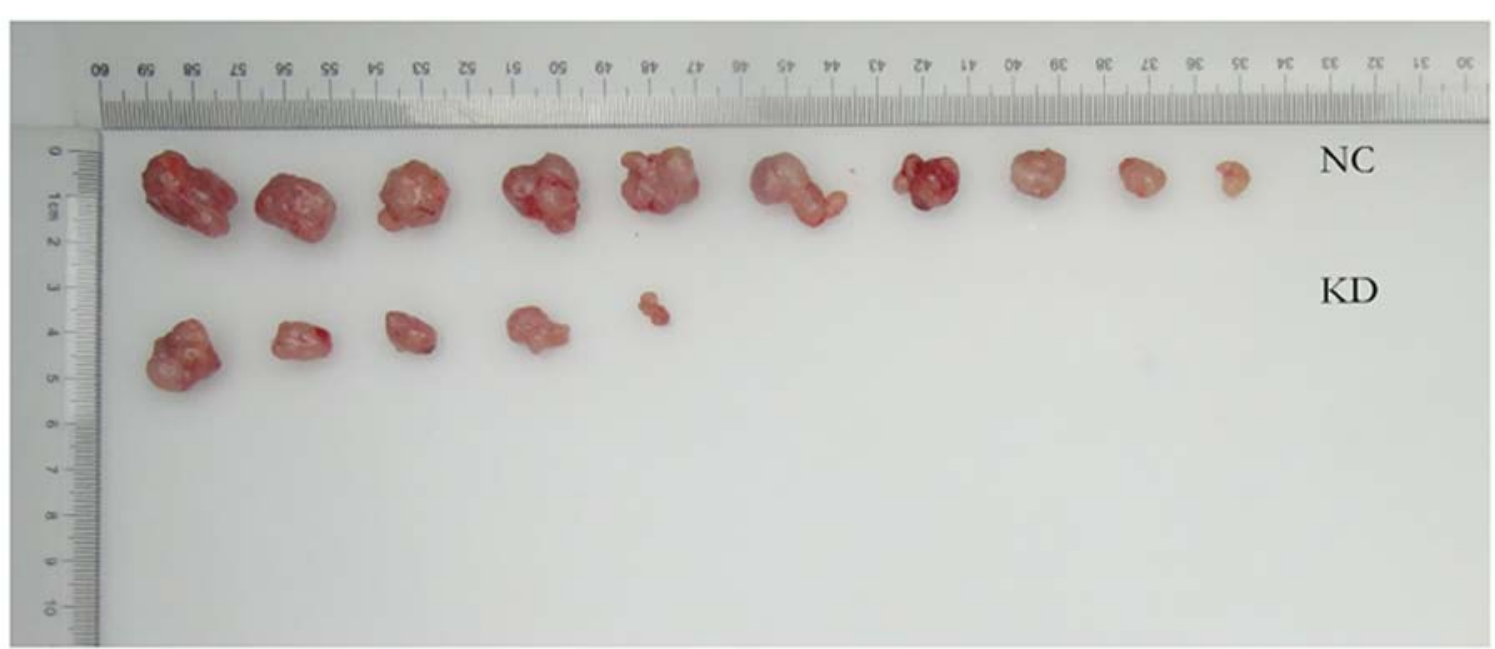

B

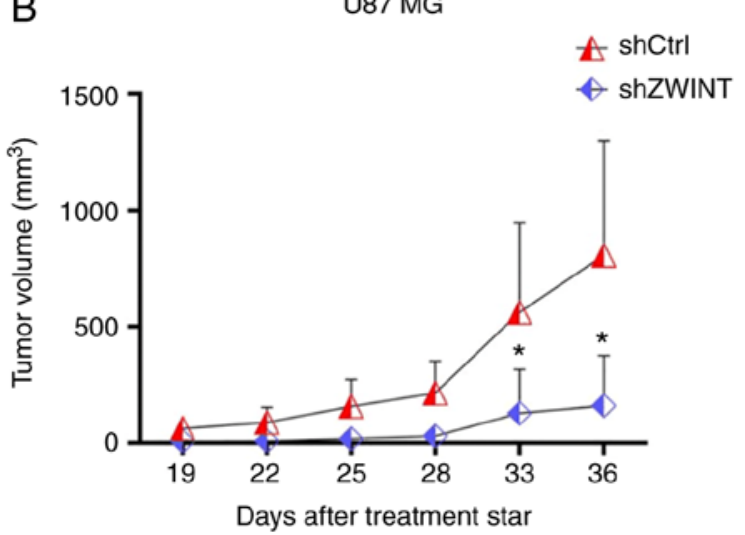

C
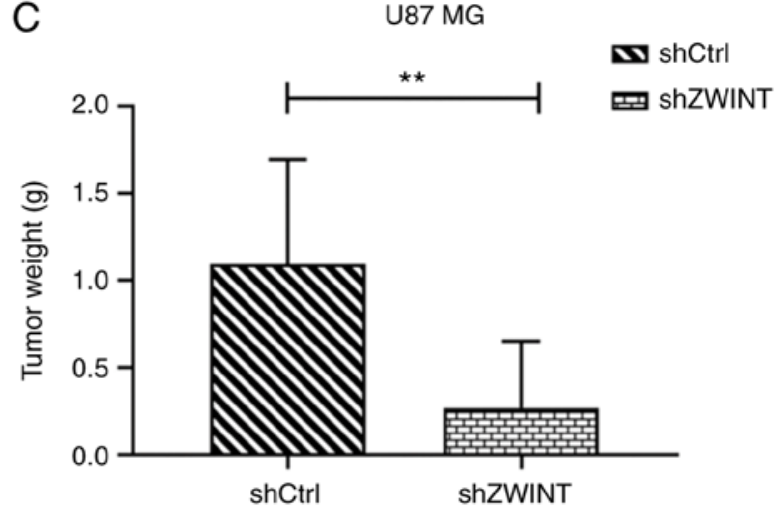

Figure 8. Effects of ZWINT knockdown on tumor growth in vivo. (A) Images of the corresponding excised tumors. (B) Growth curve of tumor volumes and $(\mathrm{C})$ histogram of tumor weights indicated that the shZWINT group tumors grew slower than the shCtrl group tumors ("P<0.05 and $\left.{ }^{* *} \mathrm{P}<0.01\right)$. ZWINT, Homo sapiens ZW10 interacting kinetochore protein.

membrane into the lower chamber was significantly lower than that in the shCtrl group. The results showed that there were fewer invading cells in the shZWINT group than that in the control group at $24 \mathrm{~h}$ after invasion, and the invasive ability was significantly inhibited $(\mathrm{P}<0.01$, Fig. 6).

Knockdown of ZWINT increases GBM cell apoptosis. In FACS analysis with Annexin-V, significantly increased apoptosis was found in the ZWINT shRNA-infected U251 and U87 MG cells from 1.57 to $11.91 \%(\mathrm{P}<0.01)$ (Fig. $7 \mathrm{~A})$. Similarly, as shown in caspase-3/7 activity analysis, the level of caspase-3/7 activity in the cells expressing shZWINT was significantly higher than that in cells expressing shCtrl $(\mathrm{P}<0.01)$ (Fig. 7B). Overall, ZWINT may have an important role in inhibiting cell apoptosis.

Knockdown of ZWINT inhibits tumor growth. To further demonstrate the role of ZWINT in vivo, we performed tumor xenograft assays. The average growth rate of the tumors in the shZWINT mouse group was significantly slower than that of the shCtrl group, and the shZWINT group had significantly decreased tumor volumes and weights compared to those of the shCtrl group (Fig. 8). The results indicated that ZWINT may act as a potent protumorigenic factor that can accelerate GBM growth.

\section{Discussion}

Currently, the molecules involved in cell cycle regulation have garnered wide attention due to their potential contribution to neoplastic transformation, and targeting cell cycle checkpoints may provide substantial improvement to malignancy therapy (27). However, there have been limited studies regarding these molecules in glioblastoma (GBM). In the present study, we first analyzed the differentially expressed genes (DEGs) between GBM and normal brain tissues from a TCGA dataset, and then ZWINT was analyzed by high-content screening. There are few data regarding ZWINT expression in tumorigenesis, especially in regards to GBM progression. This finding prompted us to perform $\mathrm{qPCR}$ and IHC to verify ZWINT expression in GBM cell lines and tissues. Our data demonstrated that ZWINT was markedly overexpressed at both the mRNA and protein levels in GBM. We further determined that interfering with ZWINT could inhibit the proliferation and invasion and accelerate the apoptosis of human GBM cell lines in vitro, and it was significantly related to the tumor growth process in vivo. Consequently, targeting ZWINT may constitute a powerful strategy for the development of novel therapies for GBM. 
Gene polymorphism is one of the biological bases of tumorigenesis, thus tumors can be considered genetic diseases. Kinetochore assembly is arguably the most critical step in determining accurate chromosome segregation, which is crucial for maintaining genomic integrity. Kinetochores are composed of a number of proteins (NDC80, MIS12, ZW10, Zwint-1, ZWILCH), which are responsible for stabilizing the kinetochore-microtubule (KMT) attachment and recruiting the components of the spindle assembly checkpoint (SAC) (28-31). ZWINT interacts with ZW10 to ensure correct chromosome motility and mitotic spindle checkpoint operation (32). ZWINT encodes a protein that is located in prophase kinetochores before ZW10 does, even remaining detectable on the kinetochore until late anaphase, and is distributed in the cytoplasm of interphase cells. We speculated that only correcting erroneous kinetochore-microtubule attachment in a timely manner and regulating spindle checkpoint function can maintain mitotic cell cycle integrity. ZWINT, as a part of the kinetochore, is required for cell proliferation and growth. Therefore, increased ZWINT causing chromosome instability can be associated with cancer progression.

In the era of 'big' data, a large number of tumor gene expression profiles have been widely used. This study made comprehensive use of various database resources and bioinformatic software to mine the DEGs during the occurrence and development of GBM to screen effective molecular targets. Functional and signaling pathway enrichment analyses were applied to identify several hub genes that interact with ZWINT to participate in the mitosis and regulation of the cell cycle of GBM cells. Tumorigenesis is a complex pathogenetic process that is driven by specific genetic and epigenetic alterations. In our present research, 6,710 DEGs were screened in total and consisted of 2,387 upregulated genes and 3,873 downregulated genes. The upregulated genes are mainly responsible for mitotic nuclear division, cell division, sister chromatid cohesion and cell proliferation, and the downregulated genes mostly participate in signal transduction, ion transport, and regulation of synaptic potential. Twenty hub genes had high degrees in the PPI network. We found that ZWINT was associated with NDC80 homolog (NDC80), serine/threonine-protein kinase PLK1 (PLK1), spindle and kinetochore associated complex subunit 1 (SKAl), which interact with each other and are mainly involved in the mitotic cell cycle, cell division, signal transduction, and AMPK signaling pathway. Abnormal gene expression or dysfunction is closely related to neoplasia.

The mitotic regulator NDC80 is highly expressed in various human malignancies, including hepatocellular carcinoma, colon cancer and osteosarcoma (33-35). NDC80 is also called $\mathrm{Hec1}$, and its complexes together with NUF2 component of NDC80 kinetochore complex (NUF2), SPC24 component of NDC80 kinetochore complex (SPC24) and SPC25 component of NDC80 kinetochore complex (SPC25) participate in the spindle assembly checkpoint and regulation of mitosis and chromosome segregation (36-39). Lin et al showed that Hecl sequentially recruits Zwint-1 and ZW10 to kinetochores during the mitotic phase of the cell cycle, and interruption of the centromeric recruitment led to chromosomal missegregation, incomplete activation of spindle examination points, and ultimately cell death (31). The above findings offer a theoretical basis for how NDC80 and ZWINT promote faithful chromosome segregation and control the spindle checkpoint.

Tripartite motif containing 17 (Terf/TRIM17) is a tripartite motif (TRIM) protein, and its coiled-coil domain is required for interaction with ZWINT. Therefore, its role in oncogenic events may be by regulating the turnover of the ZWINT protein. Endo et al found that Terf/TRIM17 exhibits E3 ubiquitin ligase activity by stimulating the degradation of the kinetochore protein ZWINT and negatively regulating cell proliferation via the proteasomal pathway in mammalian cells (5). We can use these interactions to inhibit the growth of tumor cells, and the specific molecular mechanism will be elucidated by future studies.

PLK1 and SKAI are two newly discovered genes associated with mitosis and tumorigenesis. PLK1 encodes a serine/threonine protein kinase, which is a critical regulator of cell cycle progression, cytokinesis, mitosis and the DNA damage response (40). The deletion of the PLK1 protein dramatically inhibited cancer cell proliferation and induced apoptosis (41). The SKA1 complex is a microtubule-binding subcomplex of the outer kinetochore and is essential for proper chromosome segregation (42). Previous data have shown that knockdown of SKAl inhibits cell proliferation and migration and blocks the cell cycle; moreover, inhibition of SKA1 by small-molecule inhibitors could restrain the activity of the AKT and ERK signaling pathways $(43,44)$. These studies have shown that ZWINT, NDC80, PLK1, SKA1 and Terf/TRIM17 participate in the pathogenesis of malignant neoplasms by affecting mitosis and cell cycle progression, which supports our findings.

The present research has some limitations. First, it is not sufficient to predict target genes only based on bioinformatics, and further mechanistic studies of the way ZWINT affects tumor cell proliferation, invasion and apoptosis are necessary to better understand the roles of the underlying molecule. Second, only two GBM cell lines were used in our study, and the function of ZWINT should be demonstrated in more cell lines. Finally, additional clinical information is needed to identify its prognostic value in GBM.

In summary, our preliminary research demonstrated that ZWINT may be a promising biomarker as it promotes GBM cell proliferation and invasion and inhibits apoptosis. Knockdown of ZWINT inhibited tumorigenesis in a xenograft model. Data mining and integration may be an efficient tool with which to predict the progression of GBM. The DEGs identified by comprehensive bioinformatic analyses may represent a valuable resource that may predict the progression of GBM.

\section{Acknowledgements}

Not applicable.

\section{Funding}

This study was supported by a grant from the National Natural Sciences Foundation of China (no. 81772680).

\section{Availability of data and materials}

All datasets generated during our present study are available through data mining from public gene databases. 


\section{Authors' contributions}

All the authors have made substantial contributions to this manuscript, LY, NH, and MZ were involved in the conception and design of the study. MZ guided the cell biology and animal experiments and was responsible for the whole project; XZ and YZ performed the bioinformatic data collection, integration analysis and figure processing. LY conducted the cell biology experiments; RC contributed to the animal experiments and project management; LY and NH drafted and edited the manuscript; and MZ critically revised the manuscript for important intellectual content, as well as the collection of data. All authors confirmed and approved the final manuscript.

\section{Ethics approval and consent to participate}

The animal experiments in the present study were approved by the Ethics Committee for Animal Experimentation of Tongji Hospital, Tongji Medical College Huazhong University of Science and Technology and in accordance to the institutional and university guidelines on the care and use of experimental animals.

\section{Patient consent for publication}

Not applicable.

\section{Competing interests}

The authors declare that they have no competing interests.

\section{References}

1. Liebelt BD, Shingu T, Zhou X, Ren J, Shin SA and Hu J: Glioma stem cells: Signaling, microenvironment, and therapy. Stem Cells Int 2016: 7849890, 2016.

2. Biedermann J, Preussler M, Conde M, Peitzsch M, Richter S, Wiedemuth R, Abou-El-Ardat K, Krüger A, Meinhardt M, Schackert G, et al: Mutant IDH1 differently affects redox state and metabolism in glial cells of normal and tumor origin Cancers (Basel) 11: pii: E2028, 2019.

3. Chai RC, Zhang KN, Chang YZ, Wu F, Liu YQ, Zhao Z, Wang KY, Chang YH, Jiang T and Wang YZ: Systematically characterize the clinical and biological significances of $1 \mathrm{p} 19 \mathrm{q}$ genes in $1 \mathrm{p} / 19 \mathrm{q}$ non-codeletion glioma. Carcinogenesis 40 $1229-1239,2019$.

4. Asif S, Fatima R, Krc R, Bennett J and Raza S: Comparative proteogenomic characterization of glioblastoma. CNS Oncol 8 : CNS37, 2019.

5. Endo $\mathrm{H}$, Ikeda $\mathrm{K}$, Urano $\mathrm{T}$, Horie-Inoue $\mathrm{K}$ and Inoue $\mathrm{S}$ : Terf/TRIM17 stimulates degradation of kinetochore protein ZWINT and regulates cell proliferation. J Biochem 151: 139-144, 2012.

6. Starr DA, Saffery R, Li Z, Simpson AE, Choo KH, Yen TJ and Goldberg ML: HZwint-1, a novel human kinetochore component that interacts with HZW10. J Cell Sci 113: 1939-1950, 2000.

7. Famulski JK, Vos L, Sun X and Chan G: Stable hZW10 kinetochore residency, mediated by hZwint-1 interaction, is essential for the mitotic checkpoint. J Cell Biol 180: 507-520, 2008.

8. Peng F, Li Q, Niu SQ, Shen GP, Luo Y, Chen M and Bao Y: ZWINT is the next potential target for lung cancer therapy. J Cancer Res Clin Oncol 145: 661-673, 2019.

9. Yang XY, Wu B, Ma SL, Yin L, Wu MC and Li AJ: Decreased expression of ZWINT is associated with poor prognosis in patients with HCC after surgery. Technol Cancer Res Treat 17: 1533033818794190,2018
10. Ying $\mathrm{H}, \mathrm{Xu} \mathrm{Z}$, Chen $\mathrm{M}$, Zhou $\mathrm{S}$, Liang $\mathrm{X}$ and Cai $\mathrm{X}$ : Overexpression of Zwint predicts poor prognosis and promotes the proliferation of hepatocellular carcinoma by regulating cell-cycle-related proteins. Onco Targets Ther 11: 689-702, 2018.

11. Kumar S, Clarke D and Gerstein MB: Leveraging protein dynamics to identify cancer mutational hotspots using 3D structures. Proc Natl Acad Sci USA 116: 18962-18970, 2019.

12. Ding J, McConechy MK, Horlings HM, Ha G, Chun Chan F, Funnell T, Mullaly SC, Reimand J, Bashashati A, Bader GD, et al: Systematic analysis of somatic mutations impacting gene expression in 12 tumour types. Nat Commun 6: 8554, 2015.

13. Deng M, Brägelmann J, Schultze JL and Perner S: Web-TCGA: An online platform for integrated analysis of molecular cancer data sets. BMC Bioinformatics 17: 72, 2016.

14. Johann PD, Jäger N, Pfister SM and Sill M: RF_Purify: A novel tool for comprehensive analysis of tumor-purity in methylation array data based on random forest regression. BMC Bioinformatics 20: 428, 2019.

15. Shivakumar M, Lee Y, Bang L, Garg T, Sohn KA and Kim D: Identification of epigenetic interactions between miRNA and DNA methylation associated with gene expression as potential prognostic markers in bladder cancer. BMC Med Genomics 10 (Suppl 1): S30, 2017.

16. Cooper LA, Demicco EG, Saltz JH, Powell RT, Rao A and Lazar AJ: PanCancer insights from the cancer genome atlas: The pathologist's perspective. J Pathol 244: 512-524, 2018.

17. Robinson MD, McCarthy DJ and Smyth GK: edgeR: A bioconductor package for differential expression analysis of digital gene expression data. Bioinformatics 26: 139-140, 2010.

18. McCarthy DJ, Chen Y and Smyth GK: Differential expression analysis of multifactor RNA-Seq experiments with respect to biological variation. Nucleic Acids Res 40: 4288-4297, 2012.

19. Grzmil M, Morin PJ Jr, Lino MM, Merlo A, Frank S, Wang Y, Moncayo $\mathrm{G}$ and Hemmings BA: MAP kinase-interacting kinase 1 regulates SMAD2-dependent TGF- $\beta$ signaling pathway in human glioblastoma. Cancer Res 71: 2392-2402, 2011.

20. Gautier L, Cope L, Bolstad BM and Irizarry RA: Affy-analysis of affymetrix GeneChip data at the probe level. Bioinformatics 20: 307-315, 2004.

21. Li Z, Zhao K and Tian H: Integrated analysis of differential expression and alternative splicing of non-small cell lung cancer based on RNA sequencing. Oncol Lett 14: 1519-1525, 2017.

22. Huang da W, Sherman BT and Lempicki RA: Systematic and integrative analysis of large gene lists using DAVID bioinformatics resources. Nat Protoc 4: 44-57, 2009.

23. Szklarczyk D, Franceschini A, Wyder S, Forslund K, Heller D, Huerta-Cepas J, Simonovic M, Roth A, Santos A, Tsafou KP, et al: STRING v10: Protein-protein interaction networks, integrated over the tree of life. Nucleic Acids Res 43 (Database Issue): D447-D452, 2015.

24. Franz M, Lopes CT, Huck G, Dong Y, Sumer O and Bader GD: Cytoscape.js: A graph theory library for visualisation and analysis. Bioinformatics 32: 309-311, 2016.

25. Chin $\mathrm{CH}$, Chen $\mathrm{SH}$, Wu HH, Ho CW, Ko MT and Lin CY: cytoHubba: Identifying hub objects and sub-networks from complex interactome. Bmc Syst Biol 8 (Suppl 4): S11, 2014.

26. Livak KJ and Schmittgen TD: Analysis of relative gene expression data using real-time quantitative PCR and the 2(-Delta Delta C(T)) method. Methods 25: 402-408, 2001.

27. Visconti R, Della Monica R and Grieco D: Cell cycle checkpoint in cancer: A therapeutically targetable double-edged sword. J Exp Clin Cancer Res 35: 153, 2016.

28. Cheeseman IM and Desai A: Molecular architecture of the kinetochore-microtubule interface. Nat Rev Mol Cell Biol 9: 33-46, 2008.

29. Varma D and Salmon ED: The KMN protein network-chief conductors of the kinetochore orchestra. J Cell Sci 125: 5927-5936, 2012.

30. Obuse C, Iwasaki O, Kiyomitsu T, Goshima G, Toyoda Y and Yanagida M: A conserved Mis12 centromere complex is linked to heterochromatic HP1 and outer kinetochore protein Zwint-1. Nat Cell Biol 6: 1135-1141, 2004.

31. Lin YT, Chen Y, Wu G and Lee WH: Hecl sequentially recruits Zwint-1 and ZW10 to kinetochores for faithful chromosome segregation and spindle checkpoint control. Oncogene 25: 6901-6914, 2006.

32. Woo Seo D, Yeop You S, Chung WJ, Cho DH, Kim JS and $\mathrm{Su}$ Oh J: Zwint-1 is required for spindle assembly checkpoint function and kinetochore-microtubule attachment during oocyte meiosis. Sci Rep 5: 15431, 2015. 
33. Ju LL, Chen L, Li JH, Wang YF, Lu RJ, Bian ZL and Shao JG: Effect of NDC80 in human hepatocellular carcinoma. World J Gastroenterol 23: 3675-3683, 2017.

34. Xing XK, Wu HY, Chen HL and Feng HG: NDC80 promotes proliferation and metastasis of colon cancer cells. Genet Mol Res $15,2016$.

35. Xu B, Wu DP, Xie RT, Liu LG and Yan XB: Elevated NDC80 expression is associated with poor prognosis in osteosarcoma patients. Eur Rev Med Pharmacol Sci 21: 2045-2053, 2017.

36. Matsuo Y, Maurer SP, Surrey T and Toda T: Purification and characterisation of the fission yeast Ndc80 complex. Protein Expr Purif 135: 61-69, 2017.

37. Valverde R, Ingram J and Harrison SC: Conserved tetramer junction in the kinetochore Ndc80 complex. Cell Rep 17: 1915-1922, 2016

38. Suzuki A, Badger BL and Salmon ED: A quantitative description of Ndc80 complex linkage to human kinetochores. Nat Commun 6: 8161, 2015.

39. Ferrara M, Sessa G, Fiore M, Bernard F, Asteriti IA, Cundari E, Colotti G, Ferla S, Desideri M, Buglioni S, et al: Small molecules targeted to the microtubule-Hecl interaction inhibit cancer cell growth through microtubule stabilization. Oncogene 37: 231-241, 2018.
40. Liao Y, Lin D, Cui P, Abbasi B, Chen C, Zhang Z, Zhang Y, Dong Y, Rui R and Ju S: Polo-like kinase 1 inhibition results in misaligned chromosomes and aberrant spindles in porcine oocytes during the first meiotic division. Reprod Domest Anim 53: 256-265, 2018.

41. Ma X, Wang L, Huang D, Li Y, Yang D, Li T, Li F, Sun L, Wei H, He K, et al: Polo-like kinase 1 coordinates biosynthesis during cell cycle progression by directly activating pentose phosphate pathway. Nat Commun 8: 1506, 2017.

42. Sivakumar S and Gorbsky GJ: Phosphatase-regulated recruitment of the spindle- and kinetochore-associated (Ska) complex to kinetochores. Biol Open 6: 1672-1679, 2017.

43. Zhao LJ, Yang HL, Li KY, Gao YH, Dong K, Liu ZH, Wang LX and Zhang B: Knockdown of SKA1 gene inhibits cell proliferation and metastasis in human adenoid cystic carcinoma. Biomed Pharmacother 90: 8-14, 2017.

44. Wang K, Sun J, Teng J, Yu Y, Zhong D and Fan Y: Overexpression of spindle and kinetochore-associated protein 1 contributes to the progression of prostate cancer. Tumour Biol 39: $1010428317701918,2017$.

This work is licensed under a Creative Commons Attribution-NonCommercial-NoDerivatives 4.0 International (CC BY-NC-ND 4.0) License. 